of this survey, the 18-month period ended in June 2001, the 20 physician groups almost universally did not know their actual SAI drugs costs, even for those medical groups that reported risk contracts with HMOs for these costs.

Some readers may have difficulty understanding why and how a physician group would agree to accept financial risk for a cost center, in this case SAI drugs, that is poorly measured and not clearly defined. However, a survey performed a year earlier, in 1999, by Evergreen Re, a reinsurer based in Stuart, Florida, produced results that may have predicted the results found by Agnew, Stebbins, Hickman, and Lipton. As many as 30\% of hospital and physician medical group executives in markets with considerable HMO penetration—at least 30\%—had little understanding of their level of exposure to financial risk through their capitated contracts. The hospital and medical groups in the survey reported an average 36\% of revenues from capitated contracts, and the average provider organization had capitated agreements with 5 HMOs. ${ }^{14}$

Many of the SAIs have a monthly drug cost of more than $\$ 1,000$ per patient. There is probably the temptation for some HMOs to consider the transfer of financial risk, in this case for SAIs, to physician provider groups. This will also be a short-run game if (a) the expenditures for SAIs become significant and (b) the physician groups do not have control over benefit design (e.g., out-of-pocket cost-share amount, scope of coverage, and criteria for coverage). SAI drug cost is probably best managed by a shared financial risk arrangement in which the physician groups assume a relatively small portion (e.g., 20\%) of the total financial risk and also enjoy a reasonable financial cap on losses (i.e., aggregate or patient-specific stop-loss).

\section{Evidence-based Medicine, Practice Guidelines, and Disease Management}

Early in 2003, the Agency for Healthcare Research and Quality launched the Web-based National Quality Measures Clearinghouse to function as a repository for evidence-based quality measures and measure sets. ${ }^{15}$ In January 2003, Kaiser Permanente announced that it would make available on its Web site more than 100 clinical practice guidelines (CPGs) that are used by Kaiser doctors for treatment of Kaiser $\mathrm{HMO}$ members. ${ }^{16}$ CPGs are the operational (process) part of interventions to improve clinical, service and cost outcomes. CPGs are necessary to operationalize the evidence that results from the conduct of randomized controlled trials (RCTs). Without CPGs, it is possible to systematically apply RCT evidence to real-world clinical practice. When CPGs are defined clearly and in sufficient detail, it is possible to use feedback from performance measures to continually improve care in a disease management program.

Disease management programs are difficult to design, implement, operate, and maintain, ${ }^{17}$ and, even today, there remains considerable frustration over the inability to reliably measure the financial value of disease management programs. ${ }^{18}$ In this issue of the Journal, Cannon, Larsen, Towner, et al. describe a health system- wide effort to improve clinical outcomes in diabetes. ${ }^{19}$ The authors report statistically significant and clinically important improvement in diabetes care according to 6 key performance measures: percentage of diabetics with at least 1 recorded hemoglobin Alc measurement per year, percentage of diabetics with hemoglobin Alc greater than 9.5, percentage of diabetics with hemoglobin Alc less than 7 , percentage of diabetics with at least 1 recorded low-density lipoprotein (LDL) measurement per year, percentage of diabetics with recorded LDL value less than $130 \mathrm{mg} / \mathrm{dL}$, and percentage of diabetics with at least 1 eye exam per year. The authors do not report an estimated return on investment in the diabetes care management system (DCMS) at this integrated health system.

The investment in care process models (CPMs) at Intermountain Health Care (IHC) is, in fact, large. Brent James, $\mathrm{MD}$, and his colleagues at IHC have worked for nearly 20 years to create CPMs and measure their effects on clinical and service outcomes. James is fond of saying that clinical practice improvement will result in greater efficiency and, therefore, have a favorable effect on cost outcomes as well as clinical and service outcomes.

The results of the DCMS reported in this issue of the Journal are nothing short of exciting. Yet, readers should recognize that (a) this integrated health network (IHN) has been in the business of producing, implementing, and continually improving CPMs for nearly 20 years and (b) this is not just another integrated health system. SMG Marketing, now Verispan, has found IHC to be among the top integrated health systems in the United States since it began the measurement of integrated health systems 5 years ago. As of March 1999, this IHN ranked number 15 among all IHNs in the United States. ${ }^{20}$ SMG Marketing ranked IHC number one among 532 IHNs in 1999. ${ }^{21}$ In year 2000, Sentara Healthcare (Norfolk, VA) edged out IHC as the most integrated health network in the United States by these measures, ${ }^{22}$ but IHC reclaimed the top spot in $2001^{23}$ and held on to the top spot among 472 IHNs assessed in 2002. ${ }^{24}$ IHC had about 2,000 beds among 21 hospitals in 2002, in addition to the physician division and insurance division, including IHC Health Plans.

\section{The Relative Value of Disease Management Programs Versus Drug Manufacturer Rebates}

When the State of Florida in 2001 proposed a plan to extract additional rebates from prescription drug manufacturers through imposition of a preferred drug list (PDL) tied to a priorauthorization process, selected prescription drug manufacturers made counter proposals to sponsor disease management programs in lieu of paying additional rebates. In September 2001, Florida agreed to a proposal that projected savings of $\$ 16.3$ million from establishment of 2 community-based disease management programs, one to hire health professionals and social workers to attend to Hispanic and Mexican-American Medicaid recipients with depression, HIV/AIDS, breast cancer, cervical cancer, or lung cancer. The explicit goal of this disease management program was to improve compliance with health regimens, including drug regimen adherence. ${ }^{25}$ The second disease 